\title{
Genes involved in autoimmune disease Myasthenia Gravis
}

\begin{abstract}
Myasthenia gravis (MG), a rare disorder of the neuromuscular transmission, is increasingly acknowledged as a syndrome rather than a single disease. In the recent past, there has been an active search for new antigens in myasthenia gravis, whereas clinical and experimental studies have provided new insights into crucial pathways in immune regulation, which might become the targets of future therapeutics. This review summarizes various genes involved in the pathophysiology of MG, which confirm the disease heterogeneity and may help find disease-targeted and patienttargeted therapies.
\end{abstract}

Keywords: Myasthenia, genes, AChR, MuSK, IL10, TNF
Volume 9 Issue 2 - 2019

\author{
Suhail Rasool,' Talib Khan, ${ }^{2}$ Afrozul Haq ${ }^{3}$ \\ 'Department of Neurosciences, University of California, USA \\ ${ }^{2}$ Department of Anesthesia Sheri Kashmir Institute of Medical \\ Sciences, India \\ ${ }^{3}$ Department of Food Technology, School of Interdisciplinary \\ Sciences, India
}

Correspondence: Suhail Rasool, Department of

Neurosciences, University of California, San Diego, 9500 Gilman

Dr. La Jolla, CA 92093, USA, Email suhailrasool@hotmail.com

\section{Introduction}

Myasthenia gravis (MG) is an autoimmune disorder, most often caused by pathogenic auto- antibodies (Abs) against the nicotinic acetylcholine receptor (nAChR) at the neuromuscular junction (NMJ). ${ }^{1}$ The clinical syndrome was first described by a woman initially thought to be suffering from hysteria presenting with generalized weakness, squint and dysphagia by Samuel Wilks in 1877.2 The incidence and prevalence of MG have increased over time, but this disease is still relatively rare. Globally, the prevalence of MG in most populations studied is between 100 and 200 per million, ${ }^{3}$ while the reported incidence varies widely between 1.7 and 10.4 per million. MG affects both sexes at all ages but is prevalent mostly in women during early adulthood (age $<40$ years). During puberty and older age, the incidence has been reported to be equal, ${ }^{4}$ but there are reports of higher incidence in men above the age of 50.,6 There is evidence of MG being underdiagnosed in old age ( $>80$ years). This has been attributed to variability in diagnoses in this age group, as symptoms are often interpreted as stroke or motor neuron disease. ${ }^{6}$

Genetic factors play a key role in the development and progression of MG. Up to 4 percent of patients' family members develop MG themselves, ${ }^{7}$ and there is an excess of other autoimmune diseases among family members of MG patients, ${ }^{8}$ and affected individuals themselves. ${ }^{9}$ The co-occurrence of multiple autoimmune diseases suggests shared susceptibility factors. Twin studies have shown the concordance of MG in monozygotic twins is significantly higher as compared to dizygotic twins, ${ }^{10}$ strongly suggestive of a genetic predisposition.

\section{Genetic loci associated with MG}

Several genetic loci, including both MHC and non-MHC genes, have been reported to be associated with MG (reviewed in [8 14]). HLA-A1, B8, and DR3 haplotype genes are the reproducible genetic association in early onset of myasthenia gravis (EOMG) with thymic hyperplasia. The most predominant association is with the HLA-B8 allele. ${ }^{11-14}$ A susceptibility locus, MYAS1, has been mapped to a $1.2 \mathrm{Mb}$ region comprising the distal MHC III and proximal MHC I, including tumor necrosis factor alpha (TNFA) and tumor necrosis factor beta (TNFB). ${ }^{14}$ HLA-DR7 confers a protective effect in
EOMG with thymic hyperplasia, ${ }^{15}$ with significant peaks of negative association in the TNF gene cluster and the HLA-A locus. ${ }^{8}$ For late onset of myasthenia gravis (LOMG), there are close associations with HLA-A3, B7, DR2 and HLA-DR4. ${ }^{16}$ Patients having an association with HLA-DR7 showed higher antibody titer against titin. ${ }^{15}$ Paraneoplastic MG is associated with HLA-A25, and patients with a $\mathrm{B} 2$ thymoma HLA-A2 association show a protective effect. ${ }^{17} \mathrm{~A}$ protective effect has also been suggested for the 8.1 HLA haplotype in paraneoplastic MG. ${ }^{18}$ There is an association of HLA-DR14, DQ5 with MuSK Ab-positive patients. ${ }^{19}$ The genes encoding Fc gamma receptors $(\mathrm{Fc} \gamma \mathrm{R})$ are clustered on the long arm of chromosome 1. Studies have investigated the role of functional polymorphisms in FCGR2A (encoding FcyRIIa), FCGR3A (encoding Fc $\gamma$ RIIIa) and FCGR3B (encoding Fc $\gamma$ RIIIb) are associated with MG; and it has been shown there is a high frequency of the Fc $\gamma$ RIIa $131 \mathrm{H} / \mathrm{H}$ genotype in thymoma MG patients. ${ }^{20} \mathrm{In}$ addition to that there is a high frequency of the FcyRIIa 131R/R genotype among MG patients, but with no difference between subgroups. ${ }^{21}$

Fc $\gamma$ RIIa belongs to the group of activating $F c \gamma \operatorname{Rs}^{22}$ and is found on virtually all cells of the myeloid lineage. The variant containing a histidine at amino acid position $131(131 \mathrm{H})$ has a higher affinity for $\operatorname{IgG} 2$ than the variant containing an arginine (131R). ${ }^{23}$ Because activating and inhibitory Fc $\gamma$ Rs are usually co-expressed on the cell surface and co-engaged by the $\operatorname{IgG}$ ligand, the cellular response is determined by their activation ratio. ${ }^{22}$ Thus, polymorphisms affecting receptor affinity may also modify immune responses.

\section{MuSK}

Mice lacking MuSK die at birth from respiratory failure because of the inability to form neuromuscular synapses. In the absence of MuSK all features of presynaptic and postsynaptic differentiation fail to develop, ${ }^{22}$ indicating that MuSK plays an essential role in synapse formation. AChRs -- as well as other proteins that are normally enriched at synapses-- are expressed by muscles but no longer enriched at synapses in MuSK-mutant mice, consistent with the idea that Agrin and MuSK function in the same signaling pathway. Moreover, synapsespecific transcription also requires MuSK, as 'synaptic' genes, which are normally transcribed preferentially by synaptic myofiber nuclei, are expressed similarly in all nuclei throughout muscles of MuSK- 
mutant mice..$^{22-24}$ There is convincing evidence that actin remodeling participates in clustering of AChRs. For example, stimulation of MuSK by Agrin activates Rac and Rho, which plays an important role in the formation of micro- and macroclusters of AChRs that form in cultured myotubes. ${ }^{25,26}$ Moreover, actin polymerization and regulators of F-actin assembly, such as Cortactin- Arp $2 / 3$ complex, are enriched at AChR clusters in cultured muscle cells. ${ }^{27,28}$ It remains unclear how these pathways are activated by MuSK and how actin and actin regulators act to assist in the anchoring of postsynaptic proteins. Magi-1c, a protein with a PDZ-binding domain, and two different E3 ubiquitin ligases, one containing a PDZ-binding domain, can bind to the intracellular region of MuSK..$^{29,30}$ The collagen Q (ColQ) subunit of AChE can bind to the extracellular region of MuSK, ${ }^{31}$ raising the possibility that this association, together with the strong binding between ColQ and Perlecan, an extracellular matrix protein, may assist in anchoring the ColQ form of AChE to the synaptic basal lamina.

\section{ILI 0}

IL10, the gene encoding interleukin-10 (IL-10), is located on the short arm of chromosome 1. The expression level of IL-10 in peripheral blood mononuclear cells (PBMCs) stimulated by Con A is related to three SNPs in the IL10 promoter; ${ }^{24} \mathrm{G} / \mathrm{A}$ at position -1082 , $\mathrm{T} / \mathrm{C}$ at position -819 and $\mathrm{A} / \mathrm{C}$ at position -592 . They constitute three haplotypes (GCC, ATA, ACC), which together are associated with high (GCC/GCC), medium (GCC/ATA, GCC/ACC) or low (ATA/ ATA, ATA/ACC, ACC/ACC) expression of IL-10. There are also two CA repeat microsatellites designated IL10.G and IL10.R located in the IL10 promoter. ${ }^{25} \mathrm{MG}$ patients with high titers of AChR Abs have an association to IL10.G allele 134, and MG patients with normal thymic histology have an association to IL10.R allele $112 .{ }^{26}$

\section{TNF}

TNFA (encoding tumor necrosis factor $\alpha$, TNF- $\alpha$ ) and TNFB (encoding TNF- $\beta$ ) are located in the MHC class III region, between the complement cluster and HLA-B. Two SNPs located in the promoter region of TNFA may influence transcription levels; $-308 \mathrm{G} / \mathrm{A}^{27}$ and $-238 \mathrm{G} / \mathrm{A} .^{28}$ The high expression variant TNFA -308A (designated TNFA*T2) is associated with HLA-A1, B8, and DR3, while the low expression TNFA -308G allele (designated TNFA*T1) is associated with HLA-DR4 and DR6. ${ }^{29}$ TNFB contains a NcoI diallelic restriction fragment length polymorphism in the first intron; the TNFB*1 allele correlates with increased transcription of TNF- $\beta$ compared to the TNFB*2 allele. ${ }^{30}$ Titin Ab-negative MG patients (including EOMG) have an increased frequency of the TNFA*T2 and TNFB*1 alleles, while titin Ab-positive MG patients (including paraneoplastic $\mathrm{MG})$ have an increased homozygote frequency of TNFA*T1 and TNFB*2. ${ }^{31}$ It has been observed that patients with MG with AChR antibodies with variation in the region of major histocompatibility class (MHC)II, specifically near HLADQA1/DRB1, demonstrated the strongest association signal. Association within the MHC region, albeit different loci, has been previously reported in $\mathrm{MG}^{32,33}$ In addition to MG, many other immune-mediated diseases have been associated with changes in MHC class I and II indicating the role of the $\mathrm{MHC}$ in antigen presentation. Cytotoxic lymphocyte associated protein (CTLA4) plays an important role, as an association with the locus encoding was identified. ${ }^{34}$ Further, evidence for an association with the gene for TNFRSF11A, a member of the tumor necrosis factor receptors uper family, was also detected. TNFRSF11A encodes a protein called receptor activator of NF-K $\beta$ (RANK), which increases the immune response by activation of NF-K $\beta$. The different genetic associations among MG subgroups, along with the observed clinical and pathophysiological heterogeneity, support that subgroups represent distinct etiological entities. The identification of SNPs (Single nucleotide polymorphisms) in additional genes/regions, as well as studies in African American, Hispanic, and Asian populations, are needed to move the field forward. ${ }^{35}$

\section{Discussion}

A loss of function in Agrin or MuSK results in a failure to form neuromuscular synapses, leading to respiratory failure and neonatal lethality. Despite the critical importance of these genes in synapse formation and survival, no other genes act in a redundant manner and serve as 'back-up' genes. AChRs are expressed in the postsynaptic membrane in 3- to 5-fold excess of that required for ACh to elicit an action potential and muscle contraction. CTLA4 is an immunoregulatory protein that downregulates the immune response and autoimmunity by opposing the stimulatory effect of CD28, a similar protein, and probably acts by downregulating molecules that increase T-cell reactivity, interacting with and blocking the effects of stimulatory molecules CD80 and CD86. Associations with HLADQA1/DRB1and CTLA4 were replicated in the Italian cohort. The finding of SNP singenes related to immune response is consistent with $\mathrm{MG}$ being an autoimmune disease.

\section{Conclusion}

During the last two decades there has been huge increase in number of MG patients and makes the need for better prevention and treatment even more essential. In order to obtain the valuable information about the pathogenesis and therapeutic modalities for MG, the detection of MG-specific microRNAs and the study of their functions could provide valuable information. In addition, comprehension of the interplays be-tween genes, gender, and environmental factors that might modulate disease development is highly pertinent to myasthenia. It has been investigated in MG, the frequency of SNPs in the genome is higher and they have been observed in more locations as compared to microsatellites. Therefore, clinicians and researchers focus preferentially SNPs as genetic markers. Furthermore, being easier and more robust to genotype, SNPs are automation friendly, allowing for massive interrogation in multiple candidate genes format and also in truly genomic scale studies, as it is the case in GWAS. In fact, as displayed above, association studies focus on SNPs that reside not only in exons but also in regulatory regions, such as cis-elements (promoters and operators) and splicing sites. However, the future of genetic analysis in autoimmune diseases and its corresponding applications in treatment, healthcare, and population studies lie in the rapid progress of next generation sequencing platforms which in the very next years will attain the sequencing of whole exome, transcriptome or genome, in a low-cost and accurate manner.

\section{Acknowledgments}

None.

\section{Conflicts of interest}

Authors declare no conflicts of interest.

\section{References}

1. Meriggioli MN, Sanders DB. Autoimmune myasthenia gravis: emerging clinical and biological heterogeneity. Lancet Neurol. 2009;8(5):475-490. 
2. Pearce JM. Sir Samuel Wilks (1824-1911): 'the most philosophical of english physicians’. Eur Neurol. 2009;61(2):119-123.

3. Gilhus NE. Autoimmune myasthenia gravis. Expert Rev Neurother. 2009;9(3):351-358.

4. Heldal AT, Owe JF, Gilhus NE, et al. Seropositive myasthenia gravis: a nationwide epidemiologic study. Neurology. 2009;73(2):150-151.

5. Grob D, Brunner N, Namba T, et al. Lifetime course of myasthenia gravis. Muscle Nerve. 2008;37(2):141-149.

6. Vincent A, Clover L, Buckley C, et al. Evidence of underdiagnosis of myasthenia gravis in older people. J Neurol Neurosurg Psychiatry. 2003;74(8):1105-1108.

7. Namba T, Brunner NG, Brown SB, et al. Familial myasthenia gravis Report of 27 patients in 12 families and review of 164 patients in 73 families. Arch Neurol. 1971;25(1):49-60.

8. Giraud M, Vandiedonck C, Garchon HJ. Genetic factors in autoimmune myasthenia gravis. Ann N Y Acad Sci. 2008;1132:180-192.

9. Christensen PB, Jensen TS, Tsiropoulos I, et al. Associated autoimmune diseases in myasthenia gravis. A population-based study. Acta Neurol Scand. 1995;91(3):192-195.

10. Namba T, Shapiro MS, Brunner NG, et al. Myasthenia gravis occurring in twins. J Neurol Neurosurg Psychiatry. 1971;34(5):531-534.

11. Compston DA, Vincent A, Newsom-Davis J, et al. Clinical, pathological, HLA antigen and immunological evidence for disease heterogeneity in myasthenia gravis. Brain. 1980;103(3):579-601.

12. Degli-Esposti MA, Andreas A, Christiansen FT, et al., An approach to the localization of the susceptibility genes for generalized myasthenia gravis by mapping recombinant ancestral haplotypes. Immunogenetics. 1992;35(6):355-364.

13. Janer M, Cowland A, Picard J, et al. A susceptibility region for myasthenia gravis extending into the HLA-class I sector telomeric to HLA-C. Hum Immunol. 1999;60(9):909-917.

14. Vandiedonck C, Beaurain G, Giraud M, et al. Pleiotropic effects of the 8.1 HLA haplotype in patients with autoimmune myasthenia gravis and thymus hyperplasia. Proc Natl Acad Sci U S A. 2004;101(43):15464 15469.

15. Giraud M, Beaurain G, Yamamoto AM, et al. Linkage of HLA to myasthenia gravis and genetic heterogeneity depending on anti-titin antibodies. Neurology. 2001;57(9):1555-1560.

16. Carlsson B, Wallin J, Pirskanen R, et al. Different HLA DRDQ associations in subgroups of idiopathic myasthenia gravis. Immunogenetics. 1990;31(5-6):28590.

17. Vandiedonck C, Raffoux C, Eymard B, et al. Association of HLA-A in autoimmune myasthenia gravis with thymoma. $J$ Neuroimmunol. 2009;210(1-2):120-123.

18. Spurkland A, Gilhus NE, Ronningen KS, et al. Myasthenia gravis patients with thymus hyperplasia and myasthenia gravis patients with thymoma display different HLA associations. Tissue Antigens. 1991;37(2):90-93.

19. Niks EH, Kuks JB, Roep BO, et al. Strong association of MuSK antibody-positive myasthenia gravis and HLA-DR14-DQ5. Neurology. 2006;66(11):1772-1774.
20. Raknes G, Skeie GO, Gilhus NE, et al. FcgammaRIIA and FcgammaRIIIB polymorphisms in myasthenia gravis. J Neuroimmunol. 1998;81(12):173-176.

21. van der Pol WL, Jansen MD, Kuks JB, et al. Association of the Fc gamma receptor IIA-R/R131 genotype with myasthenia gravis in Dutch patients. J Neuroimmunol. 2003;144(1-2):143-147.

22. Ravetch JV, Bolland S. IgG Fc receptors. Annu Rev Immunol. 2001;19:275-290.

23. Warmerdam PA, van de Winkel JG, Vlug A, et al. A single amino acid in the second Ig-like domain of the human Fc gamma receptor II is critical for human IgG2 binding. J Immunol. 1991;147(4):1338-1343.

24. Turner DM, Williams DM, Sankaran D, et al. An investigation of polymorphism in the interleukin-10 gene promoter. Eur J Immunogenet. 1997;24(1):1-8.

25. Eskdale J, Kube D, Tesch H, et al. Mapping of the human IL10 gene and further characterization of the 5' flanking sequence. Immunogenetics. 1997;46(2):120-128.

26. Huang DR, Zhou YH, Xia SQ, et al. Markers in the promoter region of interleukin 10 (IL-10) gene in myasthenia gravis: implications of diverse effects of IL-10 in the pathogenesis of the disease. $J$ Neuroimmunol. 1999;94(1-2):82-87.

27. Wilson AG, Symons JA, McDowell TL, et al. Effects of a polymorphism in the human tumor necrosis factor alpha promoter on transcriptional activation. Proc Natl Acad Sci U S A. 1997;94(7):3195-3199.

28. D'Alfonso S, Richiardi PM. A polymorphic variation in a putative regulation box of the TNFA promoter region. Immunogenetics. 1994;39(2):150-154.

29. Wilson AG, de Vries N, Pociot F, et al. An allelic polymorphism within the human tumor necrosis factor alpha promoter region is strongly associated with HLA A1, B8, and DR3 alleles. J Exp Med. 1993;177(2):557-560.

30. Messer G, Spengler U, Jung MC, et al. Polymorphic structure of the tumor necrosis factor (TNF) locus: an NcoI polymorphism in the first intron of the human TNF-beta gene correlates with a variant amino acid in position 26 and a reduced level of TNFbeta production. $J$ Exp Med. 1991;173(1):209-219.

31. Skeie GO, Pandey JP, Aarli JA, et al. TNFA and TNFB polymorphisms in myasthenia gravis. Arch Neurol. 1999;56(4):457-461.

32. Carlsson B, Wallin J, Pirskanen R, et al. Different HLADRDQ associations in subgroups of idiopathic myasthenia gravis. Immunogenetics. 1990;31(5-6):285-290.

33. Gregersen PK, Kosoy R, Lee AT, et al. Risk for myasthenia gravis map stoa(151) Pro $\rightarrow$ Alachange in TNIP1 and to human leukocyte antigen B*08. AnnNeurol. 2012;72(6):927-935.

34. Wang XB, Pirskanen R, Giscombe R, et al. Two SNPs in the promoter region of the CTLA4 gene affect binding of transcription factors and are associated with human myasthenia gravis. J Intern Med. 2008;263(1):61-69.

35. Renton AE, Pliner HA, Provenzano C, et al. A genome-wide association study of myasthenia gravis. JAMA Neurol. 2015;72(4):396-404. 